\title{
SDM applications to machine tools and engines
}

\author{
A SESTIERI \\ Dipartimento di Meccanica e Aeronautica, Università "La Sapienza”, Rome, \\ Italy \\ e-mail: aldo@sestieri.ing.uniroma1.it
}

\begin{abstract}
The method of structural dynamic optimization is applied here to two real-life structures, a small two-cylinder, air-cooled gasoline engine and a high speed machine tool. The aim of the first application is the reduction of the kinematic vibration transmission path composed of a piston-connecting rod, a crankshaft and an engine block, while the goal of the second application is the lowering of the peaks of the spindle-drive point FRFs measured along two orthogonal directions. It is shown how the addition of a small amount of mass at a few points on the engine block, and a small dynamic absorber with highly damped elastic connections provides the required improvements for the engine and the machine tool respectively.
\end{abstract}

Keywords. Structural dynamic modification; machining centre design; engine noise reduction.

\section{Introduction}

Two interesting applications of real systems of the frequency response-based structural modification technique are presented. The first concerns the noise reduction of an internal combustion engine, the second applies to the enhancement of the dynamic quality of a particular machine tool (a flexible manufacturing cell). The problem of the IC engine will be considered first.

Control of noise radiated by IC engines is an imperative need of the automotive industry. The problem requires a global design devoted to this goal at its preliminary stage, but this is not usually permitted because the thermodynamic and kinematic parameters as well as the main architecture of the engine and its operating conditions are considered unchangeable characteristics. Therefore, the possibility of action for the acoustician is quite limited and a reduction of even a few decibels is usually considered a successful result.

The main noise sources in an IC engine are the combustion process and the mechanical sources: piston slap which is evident at low speeds, bearing impacts and injection pump

The applications presented in this paper are drawn from: A Sestieri, W D'Ambrogio 1989 A modification method for vibration control of structures. Mech. Syst. Signal Process. 3(3): 229-253, and E I Rivin, W D'Ambrogio 1990 Enhancement of dynamic quality of a machine tool using an FRF method. Mech. Syst. Signal Process. 4(6): 495-514. Permission for using this material was obtained from Academic Press 
(for diesel engines) significant at high speeds. None of these sources can be modified, without strongly hindering the operational efficiency of the engine, as is shown by Grover $\&$ Lalor (1973), to give more than a $5 \mathrm{dBA}$ reduction in the sound radiated.

On the other hand, the sound radiated by any vibrating surface is highly dependent on the velocity and the radiation efficiency of the surface itself. Therefore, in order to achieve the goal of noise control without acting directly on either the combustion or mechanical noise sources, we may devote our strength towards the possibility of modifying the noise transmission paths through the engine structure by altering, in some way, their vibrational behaviour. In other words, once the noise radiated by the engine surfaces has been computed or measured under running conditions by means of special techniques such as selective unwrapping, multi-coherence or near-field methods (D'Ambrogio 1983), the object of the study transfers from the acoustic field to the transmission paths of vibrational energy. Such paths concern vibration transmission from the combustion chamber to the engine block and oil pan (through other elements of the engine) or vibrations generated by mechanical components.

Starting from this point, the way in which one may change the vibrational behaviour of the engine is limited to a structural modification of the elements composing the transmission path. Considering that such elements are not amenable to complex modification, the means to bring about the vibrational control can be reasonably obtained through local variations of mass, stiffness and/or damping, lumped on a limited number of positions of the path. These lumped modifications can lead to beneficial results without actually changing the main architecture of the system, especially if these modifications involve only the static elements of the path such as the engine block, engine head or oil pan.

\section{Vibration analysis of the engine}

The engine examined is a small two-cylinder, air-cooled, gasoline engine, widely used in Italy and of limited performance (about $22 \mathrm{~kW}$ at $5500 \mathrm{rpm}$ (Sestieri \& D'Ambrogio 1988).

\subsection{Preliminary analysis}

The noise radiated by the engine was measured in a reverberation chamber using one-third octave band from 100 to $10000 \mathrm{~Hz}$, and a detailed analysis was performed to estimate the amount of power radiated by the individual surfaces (D'Ambrogio 1983). The engine was divided into six surfaces and the sound power radiated by each surface was measured independently by a "near field" technique (Crocker 1981). The strongest noise source was estimated to be the right side of the block. Having identified the surface dominating the noise emission, the paths of vibration to this surface were studied on the stationary engine to determine possible means of reducing the surface vibrations and the overall radiated noise. Since cylinder pressure is usually considered one of the dominant internal noise sources, the vibration analysis is focused on the transmission paths from the head of the piston to the right-block side surface. As known, these vibrations propagate through two principal paths: a direct one and a kinematic one. The kinematic path corresponds to the piston-connecting rod, crankshaft and engine block. The direct path is through the cylinder head and the engine block. For both paths we considered the block as the most responsible element of acoustic emission, from results of previous analyses carried out on the same engine.

For studying the kinematic path, we estimated its behaviour from the experimental knowledge of the FRF of the individual components, connected in series, by means of a 


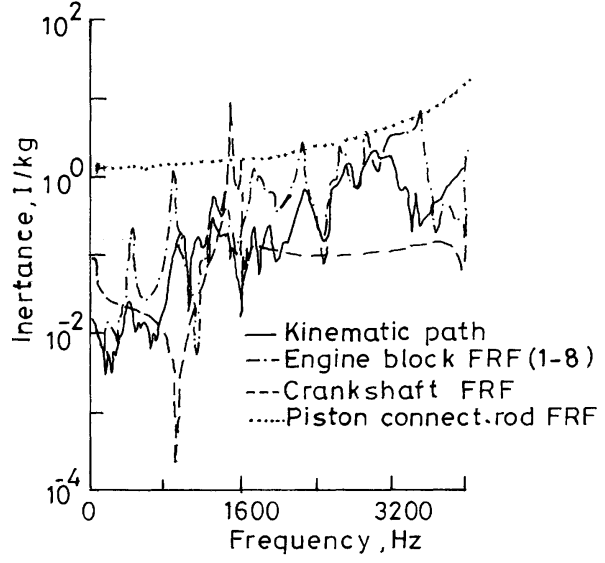

Figure 1. Kinematic path: FRF of whole path and individual components.

simple method derived from building block analysis and used by De Jong (1976). In this method only forces are accounted for, not moments. This is a reasonable statement for the kind of connections considered.

The first aim of this analysis was to identify the path component on which to perform structural modifications as those previously described (lumped masses and stiffnesses), in order to reduce the vibration transmission. Logical considerations show that the engine block is the most appropriate element to modify, since it is the sole element designed with merely static criteria. This practical indication is supported by observation of figure 1 , which compares the FRF of the individual components of the kinematic transmission path with the FRF of the assembled components. It shows that the engine block FRF is quite similar to the FRF of the whole path, whilst this is not the case for the other two components (piston-connecting rod and crankshaft). Therefore, it can be argued that the vibrational behaviour is largely controlled by the engine block, from now on identified as the component on which to act to reduce the whole response of the kinematic path to exciting vibrations. Very similar considerations might be taken for the direct path.

\subsection{Structural modification}

As a consequence of the previous considerations, a detailed study of the block was developed. Twelve points were considered on it, and the FRF matrix was experimentally determined between these points (figure 2).

These were also the points on which to locate the lumped modifications. Among them, only positions 1 and 12 (corresponding to the journal bearings) were considered not amenable to modifications.

In order to reduce the computation burden of the optimization algorithm and avoid the analyses of points having poor effects on the considered FRF, a sensitivity analysis with respect to both masses and stiffnesses was performed on the 10 points of the block. The sensitivities usually present a rapidly variable trend versus frequency, showing that, in the frequency band under consideration, single modifications of mass and stiffness alter the FRF modulus in quite a complex way. However, we can sort out modifications which do have some effect on the FRF modulus from the ones having almost no effect at all. This task could be performed by means of the RMS value of the sensitivity in the band of 


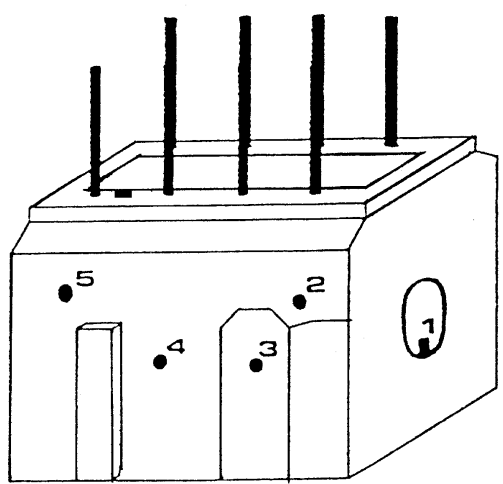

left side

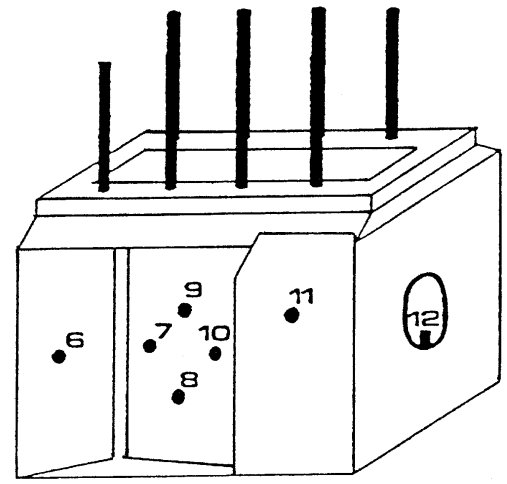

right side

Figure 2. Measurement points on the engine block.

interest, which gives a global idea of the effectiveness of a specified modification. It was observed that only points $3,4,7,8,9$, and 10 present a significant sensitivity either to mass or stiffness variations.

The FRF to change was $H_{8-1}$, with the input on the journal-1- and the response on the zone of the most seriously vibrating block -8-. At this point the optimization problem can be formulated as follows. Find

$$
\min \sum_{i \in S_{M}}\left(x_{i}\right)^{2},
$$

$x_{i}$ are the optimization variables, corresponding to mass and/or stiffness variations, $S_{M}$ is the index set of permitted modifications, with constraints,

$$
x_{i} \geq 0, \quad i \in S_{M}, \quad\left|H_{8-1}(\Delta B(x))\right| \leq\left|H_{8-1 U}\right|,
$$

in the frequency band of interest.

The frequency bands of interest here considered were two: the first one encompassed the range between 900 and $2000 \mathrm{~Hz}$, the second one $2600-3700 \mathrm{~Hz}$.

The results concerning modifications of mass and stiffness are presented in the following sections.

2.2a Mass modification: In the lower frequency band, mass modifications are effective to reduce $\left|H_{8-1}\right|$. Figure 3 compares the original FRF with results obtained by synthesis of the modified system for different values of the upper bound constraint. When decreasing this value, the reduction of the modulus improves to the detriment of the cost of modifications, as shown by the value of the objective function. In the higher bandwidth, results are better still (figure 4). This can be reasonably explained considering that the high frequency range is mass controlled.

$2.2 b$ Stiffness modification: This kind of modification does not give a significant FRF reduction in any of the examined frequency bands, although the amount of stiffness introduced is quite large. In the higher bandwidth, stiffness modification gives even more negligible results. 


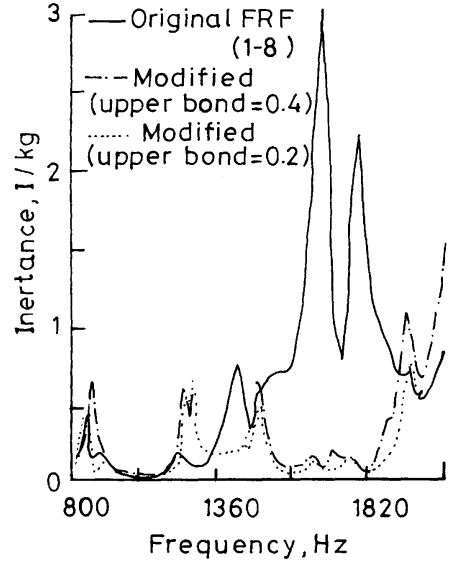

Figure 3. Original and modified FRFs for different values of the upper bound constraint.

2.2c Mass and stiffness modification: In this case too, the solution shows that the masses play the major role in both bandwidths considered. However, the modified FRF obviously reaches a lower amplitude.

2.2d Mass modification of two FRF elements: The response at a point of the block is controlled by the FRFs between the two block bearings and this point. The results obtained by synthesis on the FRF $H_{8-12}$ (between the other journal bearing and the same point 8 on the block), by modification of $H_{8-1}$ only show that a very satisfactory reduction is achieved also on the second FRF. However, since the problem of considering more constraints (relative to two or more FRFs) does not require any additional hard computation (matrix inversion), we considered the reduction of both moduli $H_{8-12}$ and $H_{8-1}$ together. The increment of the whole mass is rather low, and the single values are not too dissimilar from those computed for a single FRF.

\subsection{Experimental verification}

In order to check the computed results, the modified engine block, as determined from the mass optimization with upper modulus constraint equal to $0.4 \mathrm{~kg}^{-1}$, was experimentally

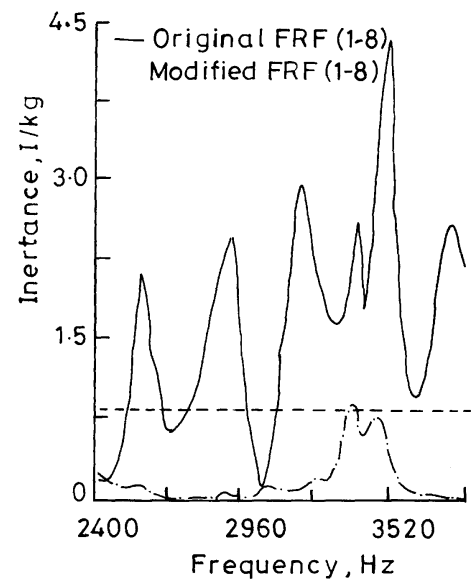

Figure 4. Original and modified FRF in the frequency band $2400-3800 \mathrm{~Hz}$. 


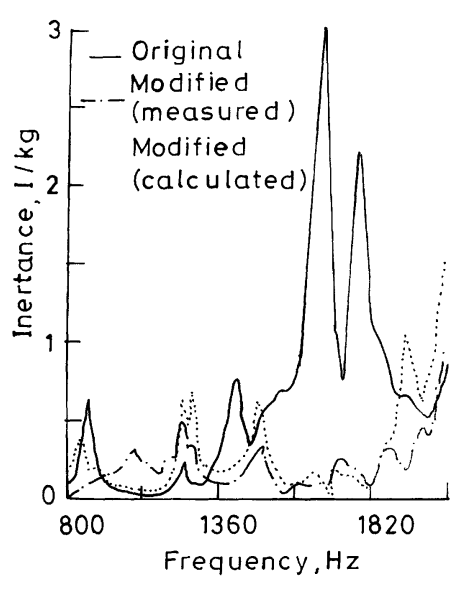

Figure 5. Comparison between computed and measured modified FRF (1-8).

tested. The prescribed values were obtained by means of lead masses bolted on the established positions of the block. Of course, these masses do not respond exactly to the scheme of lumped masses used in the computation of the inpedance matrix of the modification, $\Delta \mathbf{B}(\mathbf{x})$. The real masses have rotational inertia and present some amount of elasticity, especially with respect to their connection to the block. These effects, not considered in the computation, can change the system response in an unexpected way. However, the agreement between the computed modified system and the experimental one is quite satisfactory (figure 5), validating the effectiveness of the method and encouraging us to proceed with further developments.

In figure 6 the reduction of the direct transmission path by modification of only the kinematic path is shown. A good result is achieved for this path, confirming the correctness of choice of modification for the engine block.

It is worthwhile pointing out that the block, within the level of excitation considered, presents linear behaviour and consequently the elements of the FRF matrix are almost symmetric, as proved by preliminary tests. Therefore, in the computations, the symmetric elements, as derived from the most convenient tests carried out on the structure were indifferently used. This could explain some of the small differences observed between the experimental and numerical tests.

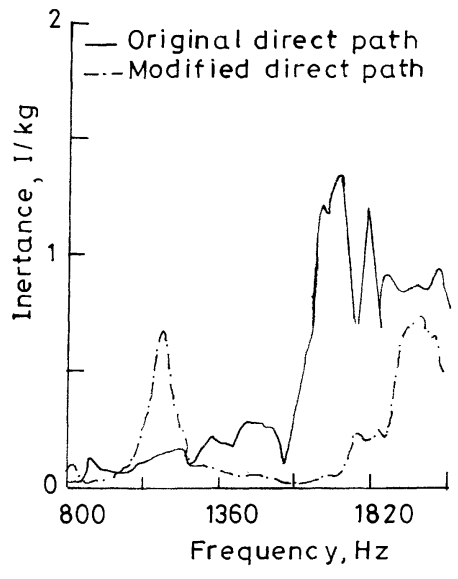

Figure 6. Original and modified direct transmission path as a by-product of the changes imposed on the kinematic path. 


\subsection{Conclusions}

A method to change the vibrational behaviour of an engine is presented here. If the combustion and/or or mechanical forces are determined or measured and the radiation efficiency is known, the method can be successfully used to control the noise. The method finds an optimal structural solution in a permitted class of modifications, restricted to lumped variations of mass, stiffness and damping. Since the engine is a very complex system, its behaviour is experimentally determined. Starting from these measured data, a modification is performed on a specific component of the vibrational energy paths. In fact, the engine presents the advantageous circumstance that the part of the system (the engine block) radiating the most is also the most suitable element on which to perform modifications, either because it is a static element or because it is large enough to allow several modifications.

On the other hand, the engine presents high modal density which makes modal identification difficult to work with. Consequently, modal synthesis processes, and sensitivity analysis or structural modifications, are very difficult to fulfill with techniques using modes of vibration. On the contrary, modal problems do not affect the optimization procedure, because the method uses only experimental raw data. The computed results are quite satisfactory and the experimental tests carried out on the modified system agree very well with the results derived by synthesis of the modified model.

\section{Structural modification applied to a machine tool}

This test was performed on a Flexible Manufacturing Cell (FMC) at General Motors (Michigan, USA) and was useful to test the validity of the structural modification technique on a large complex structure with large structural joints (Rivin \& D'Ambrogio 1990).

\subsection{Description of the machine}

The machine in figure 7 has its base elastically mounted on the floor; $X$-slide movably supported by the base; $Y$-slide movably supported on the $X$-moving slide; spindle housing sliding in $Z$-direction relative to the supporting $Y$-moving slide; the spindle located inside the spindle housing (maximum speed 15,000 rpm). Each sliding motion is serviced by an independent electric motor, coupled with the moving unit via a ball-screw.

\subsection{Evaluation of dynamic behaviour of the machine}

The structural modification approach requires the knowledge of transfer functions between many locations of the machine. Since the use of hydraulic or electrodynamic exciters on this particular machine is feasible only in a few locations (due to space limitations), applicability of impact hammer excitation technique was first verified.

The assessment of dynamic stability of a machine tool is usually a very demanding task. However, the highest peaks of spindle frequency responses are usually an indication of potential problems, both chatter-related and forced-vibration related. Spindle-drive point inertance plots showed the highest frequency response amplitudes in the frequency range $200-250 \mathrm{~Hz}$. Even if the inertance was double-integrated to get the compliance, which is directly related to the machine accuracy, the highest peak outside the low frequency range was observed at $250 \mathrm{~Hz}$, within the spindle rpm range. 

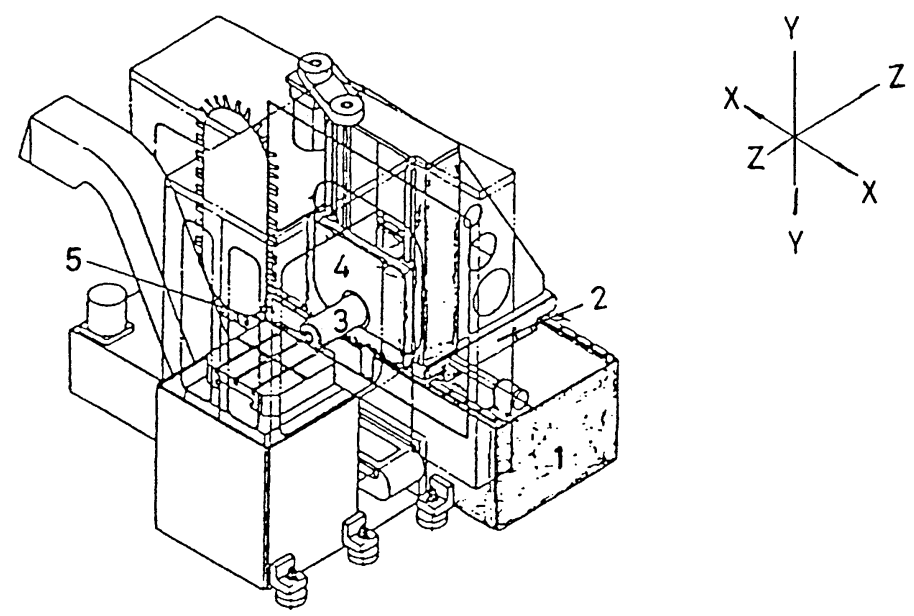

Figure 7. Sketch of the FMS: 1, base; 2, $X$-moving slide; 3, $Y$-moving slide; 4, spindle housing; 5 , spindle.

Consequently the following targets for improving the dynamic behaviour of the machine were specified.

- Lowering the peak of the spindle-drive point FRF measured in the $Y$-direction, at a frequency of about $250 \mathrm{~Hz}$, corresponding to $15,000 \mathrm{rpm}$;

- Lowering the peaks of the spindle drive point FRF measured in the $X$-direction, at frequencies of about $195 \mathrm{~Hz}$, corresponding to $11,700 \mathrm{rpm}$ and of about $220 \mathrm{~Hz}$, corresponding to $13,200 \mathrm{rpm}$.

$3.2 a$ Selection of measurement points: The selection process of locations for performing measurements requires great care. All the information about the structure dynamics is contained in the measured FRF matrix. Of course, the analyst must establish which points and which degrees of freedom have to be included in the FRF matrix. It is a crucial decision since a structural modification involving a given point or a given DOF can be evaluated only if that DOF is included in the FRF matrix. So, once the FRF matrix has been determined among a set of points and DOFs the further process will regard only the evaluation and the optimization of structural modifications involving that set of points and DOFs.

Moreover, the measured FRF matrix must contain the FRF elements which are considered the ones requiring improvement, although structural modifications may not involve DOFs associated with such FRF elements.

Therefore, deciding how many and which points have to be measured is a trade-off between two opposite requirements - to consider more and more DOFs in order to get more effective and optimally located structural modifications, or to lower the number of DOFs to reduce the experimental work and avoid the problem of storing and processing huge amounts of data.

In the case discussed, the purpose was to determine some locations where modifications would be feasible and effective without hindering normal machine operation. Eight points at the back of the machine were selected, considering only one direction per point (usually the direction normal to the surface). A possibility that some modifications, performed in locations at the back of the machine, could positively affect the FRFs on the spindle, had to 
be explored since these locations are far from the work zone and their modification the least objectionable. Two cross-sections were selected on the spindle housing: 3 DOFs were considered for the front plane (where the FRF had to be controlled) and 2 DOFs for the cross-section in the middle, that was regarded as amenable to modifications. Overall, the FRFs for 13 DOFs corresponding to a total of 91 FRF measurements, had to be determined (refer to Rivin \& D'Ambrogio 1990 for the figures).

\subsection{Structural modification of the machine}

3.3a Sensitivity analysis: This was performed in order to provide a first estimate of effectiveness of each modification in lowering the spindle response. This helps to reduce the computer time spent at the optimization stage; the results of sensitivity analysis are used to eliminate the less effective modifications from the set of optimization variables.

The sensitivity analysis was performed by considering effects of adding a single mass of increasing magnitude to each of the selected points. Dynamic absorbers, whose chatter suppressing characteristics are generally well-known, were also considered. Stiffeners or viscous damping attachments were not considered because their implementation usually requires external supporting structures, and this was not feasible in this case.

At first, effects of adding masses to the back of the machine were examined. It was found, as the result of this sensitivity analysis, that modifications of the back of the machine have only a very slight effect on the spindle-related FRFs. The amounts considered varied between 0 and $10 \mathrm{~kg}$, with a step of $1 \mathrm{~kg}$.

Then the effect of adding masses on the spindle housing was examined. The effect was not encouraging. The amounts considered varied between 0 and $5 \mathrm{~kg}$, with a step of $0.5 \mathrm{~kg}$. The main peak in $Y$-direction was significantly increasing with the increasing mass, while the main peak in $X$-direction was slightly decreasing.

Finally, the effect of a dynamic absorber on the spindle housing was examined. A twodirectional absorber - i.e. an absorber acting on both $X$ and $Y$-direction - with a tuning frequency of about $250 \mathrm{~Hz}$ was considered. The mass value was set equal to $2 \mathrm{~kg}$, and consequently the stiffness values in both $X$ and $Y$-directions were of $5 \cdot 10^{6} \mathrm{~N} / \mathrm{m}$, while the loss factor was set equal to 0.2 . In this case a significant improvement in the spindle FRFs was achieved.

\subsection{Application of the optimization algorithm}

3.4a Selection of objectives: The investigation was limited to two of the previously stated objectives: to lower the $X$-response peak at about $220 \mathrm{~Hz}$ and the $Y$-response peak at about $250 \mathrm{~Hz}$.

The first target was to improve the $X$-direction FRF on the spindle in the frequency range between 187.5 and $237.5 \mathrm{~Hz}$. In this frequency range the amplitude of the frequency response was required to be lower than the upper bound set equal to $0.1 \mathrm{~kg}^{-1}$. This involves a reduction of about $40 \%$ for the peak at $220 \mathrm{~Hz}$, and a reduction of about $25 \%$ for the other two peaks around $200 \mathrm{~Hz}$. The problem was formulated as follows: find

$$
\min _{\mathbf{x}} \sum_{i=1}^{N} \mathbf{w}_{i} x_{i}^{2},
$$


with constraints

$$
\left|H_{i j}\left(\mathbf{x}, \omega_{k}\right)\right| \leq p_{U}\left(\omega_{k}\right), \quad k=1, \ldots, N_{F},
$$

and

$$
0 \leq x_{i} \leq x_{i}^{U}, \quad i=1, \ldots, N,
$$

where $\mathbf{w}_{i}$ is a weighting vector allowing the specification of a different cost for each modification, $\mathbf{x}$ is the vector of structural modifications (optimization variables), $x_{i}^{U}$ is the upper limit for the $i$ th modification, $N$ is the number of optimization variables, $\omega_{k}$ is the $k$ th angular frequency, $N_{F}$ is the discrete number of frequencies used to represent the frequency range of interest, $H_{i j}\left(\mathbf{x}, \omega_{k}\right)$ is the FRF element to be improved, and $p_{U}\left(\omega_{k}\right)$ is the upper bound for $\left|H_{i j}\left(\mathbf{x}, \omega_{k}\right)\right|$.

3.4b Mass addition: The specified objective was first pursued by allowing mass additions on the spindle housing and at the back of the machine. A computer run of the optimization code was performed with the previously stated constraints, and with the optimization variables $\mathbf{x}$ corresponding to mass additions on DOFs 4, 5 and 8 .

The result of this run confirmed results of the sensitivity analysis, namely that the spindle responses are relatively insensitive to addition of masses at the back. Modifications at the back were made extremely desirable by an appropriate assignment of weighing factors: the cost of modification at the back was assigned to be ten times lower than the cost of modifications on the spindle housing, while a starting value of $2 \mathrm{~kg}$ on the spindle and $20 \mathrm{~kg}$ on the back was given to $x$. Nevertheless, at the end the mass value on spindle housing had increased up to $10 \mathrm{~kg}$ (the upper limit set up for this mass) while the mass value on point $8 \mathrm{had}$ decreased down to $9 \mathrm{~kg}$ (the upper limit set for this mass was $100 \mathrm{~kg}$ ).

Moreover, these values did not represent an optimal solution. The amplitude of the modified FRF was still much higher than the specified upper bound of $0.1 \mathrm{~kg}^{-1}$. In fact the optimization routine was terminated only because the solution point could not be further improved and not because an optimal solution had been found.

3.4c Dynamic absorber addition: The same goal - the improvement of the $X$-direction FRF - was pursued by allowing a two-directional $(X-Y)$ dynamic absorber to be placed on the spindle housing, with an a priori selected loss factor of 0.2 , that seems to be a reasonable average value. The loss factor was not included among the optimization variables, because its value is very hard to control if the absorber has to be designed and built for an experimental test.

In this case the result showed a significant improvement of the modified FRF with respect to the original one in the frequency range covered by the optimization (see figure $8 \mathrm{a}$ ). The modification values at the final point of the optimization procedure were as follows:

mass of the absorber $\quad 2.09 \mathrm{~kg}$ stiffness on $X$-direction $4.14 \times 10^{6} \mathrm{~N} / \mathrm{m}$ stiffness on $Y$-direction $4.14 \times 10^{6} \mathrm{~N} / \mathrm{m}$

The effect of this modification on the $Y$-direction FRF on the spindle was computed. Its improvement as a by-product of improving the $X$-direction FRF is also significant, as shown in figure $8 \mathrm{~b}$. Due to the significant improvement of the $Y$-direction FRF as a 
(a)

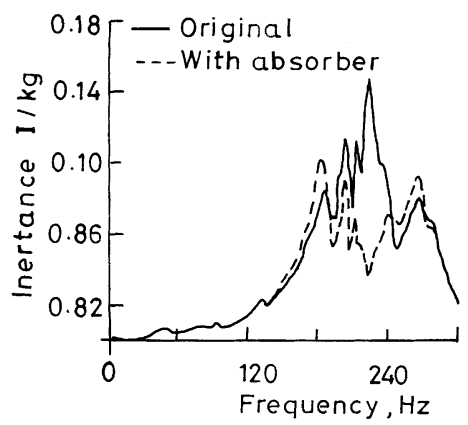

(b)

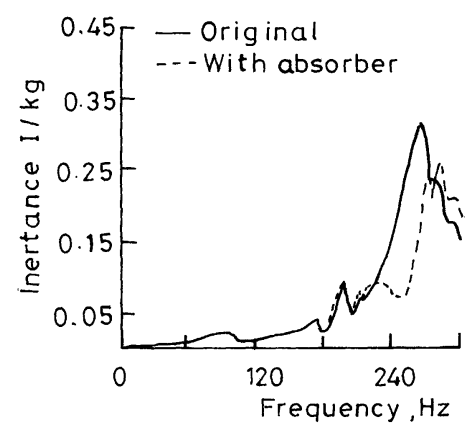

Figure 8. Original and modified spindle drive point inertance (optimization of dynamic absorber): - original, - - - with absorber (a) $X$ direction, (b) $Y$ direction.

consequence of the above modification, no need was recognized to consider an additional optimization problem in order to specifically improve the $Y$-direction FRF.

3.4d Sensitivity to loss factor: A parametric analysis was conducted to explore the effect of varying the loss factor between 0.1 and 0.4 , while keeping mass and stiffness of the absorber to the above specified values. The purpose of such analysis was to determine how critical the damping value in the practical construction of the absorber is. Although some differences appear, it was observed that they were not very significant.

\subsection{Experimental verification}

The effectiveness of attaching the dynamic absorber on the spindle housing as recommended by the optimization algorithm had to be tested. In order to avoid interference during the $Z$-direction motion of the spindle housing, the correct position for a permanent placement of the absorber should be the inside of the spindle housing, using available space between the spindle and the housing (figure 9). However to be able to conduct dynamic tests with the machine in non-operating conditions, the prototype absorber was located on the outside of the spindle housing, and thus was directly accessible for the necessary adjustments. Experimental FRF measurements were performed using impact excitation with the same force and acceleration transducers used previously.

3.5a Design of the absorber: The absorber was designed as a ring-shaped steel mass of the prescribed value, connected to the outside of the housing by means of four elastomeric

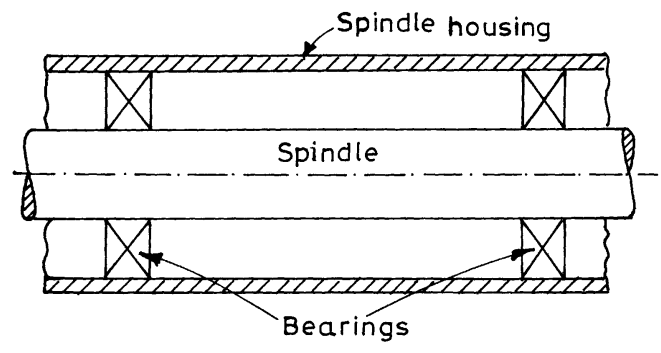

Figure 9. Sketch of the spindle housing. 

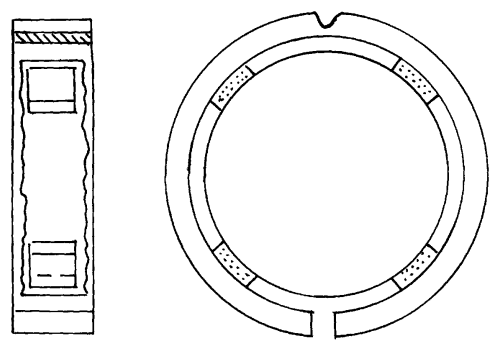

Figure 10. Sketch of the dynamic absorber.

pads providing resilience and hysteretic damping. As shown in figure 10, a cut on one side and a notch on the opposite one were designed to facilitate assembly and disassembly of the absorber. While it was easy to get the correct value for the mass of the absorber, this was not the case for stiffness and damping. It was almost impossible to size the pads in a reliable way at the design stage.

Elastomers were chosen as materials for the elastic members. Proper selection of a elastomer blend assures a combination of relatively high damping, which is needed in this application, together with required stiffness values. However, rubber is a very singular material and its properties vary as functions of load, frequency, shape, boundary conditions, temperature and time. For the available types of rubber, only limited experimental data were available on frequency dependence of the complex stiffness of the compressed samples. Due to the rigid time constraints of this project, it was impossible to perform a comprehensive evaluation of various rubber components. Thus, all the available data were used, together with theoretical expressions for the static stiffness of rubber in compression (Gent \& Meinecke 1970; Lavendel 1976) to design the rubber pads.

3.5b Tests with the absorber: Due to uncertainties inherent to the absorber design, the tests were scheduled so that a 'cut-and-try' process could be performed with the rubber pads, in order to get the correct stiffness value. Two types of elastomeric materials were used, that will be referred as 'black' elastomer and 'blue' elastomer.

\subsection{Discussion of results}

The results showed some lack of repeatability, in spite of the efforts spent to reproduce the same testing conditions. The problem did not lie in the measurement chain. So, whatever reason causes a lack of repeatability, it was ascribed to the absorber itself. As a matter of fact, mounting the absorber on the spindle housing was not easy. The rubber pads had to be forced in their position by using the elasticity of the steel ring. In addition, the external surface of the spindle housing was usually soiled because of a leak of cooling liquid. Both situations certainly affected the boundary conditions of the rubber pads: they are perhaps responsible for the discrepancies noticed between the different tests, that were always performed after removing and remounting the absorber.

However, the amplitude reductions of the spindle FRFs were significant. This demonstrated that the optimization technique based on the frequency response is effective also on complex structures like machine tools, since the dynamic quality of this machine can be effectively improved by using a vibration absorber with the specified characteristics. 


\subsection{Conclusions}

It was shown that the method of structural dynamic modification without modal identification of the mathematical model can be successfully used for such complex systems as machine tools; application of the method does not require arbitrary assumptions and does not exhibit ambiguities resident in the modal approach.

Structural modification by using masses, even attached to a relatively light spindle, may not be effective (it is highly dependent on the machine architecture), while use of stiffness either between various points on the structure or between the considered and supporting structure does not seem to be practical for machine tools.

Damped dynamic absorbers have a very strong effect even with small inertia masses and not very precise tuning; their effect spreads over broad frequency intervals thus demonstrating a relatively low sensitivity to tuning accuracy.

\section{References}

Crocker M J 1981 The use of existing and advanced intensity techniques to identify noise source on a diesel engine. SAE paper 810694

D’Ambrogio W 1983 Comportamento strutturale acustico di un motore a combustione inertia bicilindrico. Caratterizzazione acustica. Masters thesis, University di Roma, Rome

De Jong R 1976 Vibrational energy transfer in a diesel engine. Ph D thesis, MIT, Boston, MA

Gent A N, Meinecke E A 1970 Compression, bending and shear of bonded blocks. Polym. Eng. Sci. 10: $48-53$

Grover E C, Lalor N 1973 Review of low noise engine design at ISVR. J. Sound Vibr. 28:

Lavendel E E 1976 Analysis of engineered rubber elements (Moscow: Mashinostroenie)

Rivin E I, D'Ambrogio W 1990 Enhancement of the dynamic quality of a machine tool using a frequency response optimization method. Mech. Syst. Signal Process. 4: 495-514

Sestieri A, D'Ambrogio W 1988 Altering the vibrational behaviour of an IC engine as a preliminary step for noise reduction. Proc. Recent Advances in Structural Dynamics 